\title{
Morbilidad y mortalidad por cáncer oral y faríngeo en Chile*
}

\author{
Paula Riera $\mathbf{S}^{\mathbf{1}}$, Benjamín Martínez $\mathbf{R}^{\mathbf{2}}$. \\ Morbidity and mortality for oral \\ and pharyngeal cancer in Chile
}

Background: Most oral cancers are squamous cell carcinomas (90\%) which are two to four times more common in men than in women. The reasons for these differences are associated with exposure to factors such as tobacco and alcohol. Age is also considered as a risk factor (about $90 \%$ of the cases are diagnosed after 45 years of age). Aim: To analyze the frequency of oral cavity cancer during the last years in Chile. Material and methods: Mortality rates were obtained from death records of the "Instituto Nacional de Estadísticas" and publications of the World Health Organization, from 1955 to 2002. Morbidity from 1969 to 2002 was obtained from hospital discharge records of the Chilean Ministry of Health. Results: Oral cancer corresponded to $1.6 \%$ of total cancer cases in Chile, with a male:female ratio of 2.3 to 1 . Deaths due to oral cancer was $1 \%$ of all cancer deaths, with a male:female ratio of 2.8 to 1 . The morbidity rate for both genders increased while the mortality rate was relatively constant. However, we observed an increase in the mortality rate among women from 1980 to 2002, associated with more than 100\% increase in the frecuency of smoking, between 1970 and 1998. The most common anatomical location was the tongue. Conclusions: The incidences of oral cancer is increasing in Chilean women, but men are more commonly affected (Rev Méd Chile 2005; 133: 555-63).

(Key Words: Mouth neoplasms; Oropharyngeal neoplasms; Pharyngeal neoplasms)

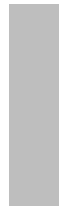

Recibido el 26 de agosto, 2004 . Aceptado en versión corregida el 23 de marzo, 2005.
"Trabajo presentado parcialmente como requisito para obtener el título de Cirujano Dentis-
ta por Paula Riera S., en la Facultad de Odontología, Universidad Mayor.
Facultad de Odontología, Universidad Mayor. Santiago de Chile.
${ }^{1}$ Egresada de Odontología, 6o ${ }^{\circ}$ año. Universidad Mayor.
${ }^{2}$ Cirujano Dentista

$\mathrm{E}^{\prime}$ cáncer oral más común es el carcinoma espinocelular (90\%), otros tipos corresponden a melanoma, linfoma, sarcomas, carcinomas de glándulas salivales menores y metástasis ${ }^{1}$. El cáncer oral es entre dos a cuatro veces más frecuente en

Correspondencia a: Dr. Benjamín Martínez. Facultad de Odontología, Universidad Mayor, Alameda Bdo. O'Higgins 2013, Santiago, Chile. Fax: 4205700. E mail: bemaro1@yahoo.com

hombres en la mayoría de los grupos étnicos, excepto en Filipinas, donde los porcentajes son similares $^{2}$. La razón para estas diferencias no están claramente establecidas, se piensa que la exposición a factores de riesgo no es igual en ambos géneros, y dentro de éstos, se ha demostrado que el uso de tabaco tiene un efecto carcinogénico directo sobre el epitelio de la cavidad oral; su asociación con alcohol, aumentaría el riesgo, entre 6 a 15 veces $^{3}$. Ha adquirido importancia en la posible etiología del 
cáncer oral la infección por virus, entre ellos el virus herpes simple (VHS-1), y el papilomavirus humano 16 (PVH 16) ${ }^{1}$. En un estudio reciente, realizado por Gillison y cols $(2000)^{4}$, encontraron parte del genoma viral en las células tumorales, pero aún no existe acuerdo con respecto al rol de virus en carcinoma espinocelular de la boca.

La edad también se considera un factor de riesgo, 90\% son diagnosticados en pacientes de más de 45 años, sin embargo ha aumentado el número de adultos entre 20 y 30 años que han desarrollado cáncer, especialmente en lengua 5 .

El cáncer faríngeo es poco común en muchos países, cáncer nasofaríngeo y de faringe inferior presentan alta incidencia en China y en el sur de Asia, tasa de 10,8 por cada 100.000 habitantes. El principal factor de riesgo que se ha identificado, está relacionado con la dieta en los asiáticos (pescado salado), y se cree que el virus EpsteinBarr también tendría un rol importante, pero aún se está estudiando ${ }^{6}$, y es factor de riesgo al igual que en el cáncer oral el excesivo consumo de tabaco y alcohol ${ }^{7}$.

Los datos de morbilidad y mortalidad representan herramientas para evaluar el riesgo de muerte en una población y la repercusión de las enfermedades en la salud, también la gravedad de las enfermedades y la sobrevida experimentada por la población. Como tales, son herramientas esenciales en el análisis de la situación de salud, la vigilancia en salud pública, la programación y la evaluación de programas y políticas de salud ${ }^{8}$.

La morbilidad y mortalidad por cáncer oral es diferente para cada país. Este trabajo pretende dar a conocer cuál es la realidad chilena con respecto al cáncer oral y faríngeo y cómo ha sido su tendencia en los últimos años.

\section{Material y MÉtodo}

Los datos de mortalidad fueron recolectados en el Instituto Nacional de Estadística (INE) a través de los registros de defunciones, por tipo de certificación y grupo de edad, según lista detallada de causas de muerte y sexo para los años 1982 a 2002, de acuerdo a los códigos de la Clasificación Internacional de Enfermedades (CIE), para los años 1982-1995, CIE-9: 140-208; los años posteriores corresponden a los códigos CIE-10: C00 al
C97. Estos datos fueron ordenados y agrupados en tablas por género y grupos de edades: 0-4, 5-9, 10-14, 15-24, 25-34, 35-44, 45-54, 55-64, 65-74 y más de 75 años.

Los datos de mortalidad para todos los cánceres, cáncer oral y faríngeo, desde 1955 a 1994, y sus respectivas tasas, fueron obtenidos de las publicaciones que realiza la Organización Mundial de la Salud (WHO) en su página de Internet (www-depdb.iarc.fr/who/menu.htm).

De los datos de mortalidad por cáncer oral, fueron seleccionadas y agrupadas las siguientes ubicaciones anatómicas:

Labio: CIE- 9: 140; CIE-10: C00; Lengua: CIE-9: 141; CIE-10: C01 y C02; Encía: CIE-9: 143; CIE-10: C03; Piso de boca: CIE-9: 144; CIE-10: C04; Glándulas salivales mayores: CIE-9: 142; CIE-10: C07 y C08.

Los datos de mortalidad correspondiente a 1993 no fueron incluidos en el estudio por presentar discrepancias ${ }^{8}$. Los datos de morbilidad se obtuvieron del Ministerio de Salud, sobre egresos hospitalarios, por grupo de edad según causa de morbilidad, para los años 1969 a 2002, lamentablemente sólo fueron publicados por el Ministerio de Salud los datos de 1969, 1970, 1971, 1973, 1975, 1981 a 1985, 1987 a 1993, 1996, 2001 y $2002^{9}$.

Los años 2001 y 2002 no fueron analizados, ya que los registros están publicados en grupos de edades que no coinciden con el resto del estudio (04, 5-9, 10-19, 20-44, 45-64, +65 años). Los datos de población se obtuvieron de los Anuarios de Demografía que publica el INE, en su registro de población total estimada al 30 de junio de cada año, por área urbana-rural y sexo según grupos de edad ${ }^{10}$. Con los datos, fueron calculadas las tasas brutas por 100.000 habitantes, se obtuvieron porcentajes para comparar morbilidad y mortalidad por cáncer oral y faríngeo con respecto a todos los cánceres.

Estadística. Análisis de regresión simple y correlación de Pearson (r), mediante el software estadístico Systat $10.2^{11}$.

\section{Resultados}

Mortalidad. La mortalidad, entre 1955 y 2002, por cáncer oral y faríngeo, representó alrededor de 1\% 
con respecto a todos los cánceres (CIE: 9 140-208; CIE: 10 C00-C97). La tasa bruta de mortalidad por cáncer oral y faríngeo, entre 1955 y 2002, fue en aumento de 0,9 a 1,3 por 100.000 habitantes (variación anual: 28,7\%). La mayor alza ocurrió en el período de 1955 a 1980, con crecimiento de $49 \%$ anual ( $r=0,702)$; en cambio desde 1981 a 2002, se mantuvo constante, existiendo incluso leve disminución ( $\mathrm{r}=-0,013)$ (Figura y Tabla 1 ). Al analizar la tendencia por género para el mismo período de años, en hombres, observamos un aumento de la tasa: 1,1 a 2,1 por 100.000 habitantes $(18 \%$ anual), el mayor incremento ocurrió de 1955 a 1980 (37\% anual, $r=0,613)$; en cambio, de 1981 a 2002, disminuyeron las tasas en 6,6\% anual $(\mathrm{r}=-0,285)$ (Figura 2 y Tabla 1$)$. En mujeres, desde 1955 a 2002, se observó disminución en la tasa de sólo 0,8\% anual, esta disminución ocurrió entre 1955 a 1980 (2,5\% anual $\mathrm{r}=-0,15)$; en cambio, desde 1981 a 2002 aumentó la tasa en 17\% anual $(\mathrm{r}=0,415)$ (Figura 3 y Tabla 1$)$.

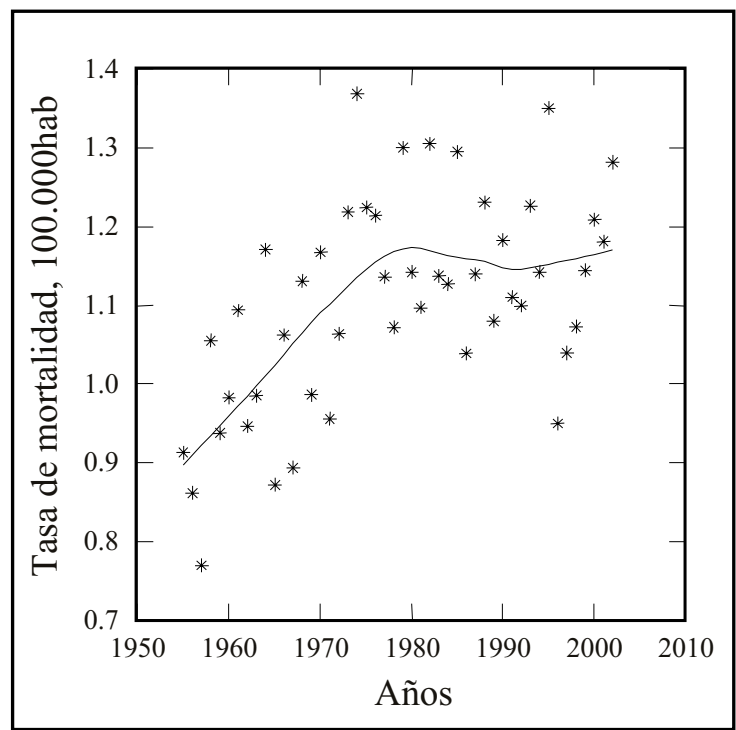

FiguRa 1. Tasa bruta de mortalidad para cáncer oral y faríngeo, ambos géneros. 1955-2002.

Tabla 1. M ortalidad por cáncer oral y faríngeo, tasas brutas de mortalidad, C hile 1955-2002

\begin{tabular}{|c|c|c|c|c|c|c|c|c|c|c|c|c|c|}
\hline \multirow[b]{2}{*}{ Año } & \multicolumn{2}{|c|}{ Hombres } & \multicolumn{2}{|c|}{ Mujeres } & \multicolumn{2}{|c|}{ Total } & \multirow[b]{2}{*}{ Año } & \multicolumn{2}{|c|}{ Hombres } & \multicolumn{2}{|c|}{ Mujeres } & \multicolumn{2}{|l|}{ Total } \\
\hline & Defunciones & Tasa & Defunciones & Tasa & Defunciones & Tasa & & Defunciones & Tasa & Defunciones & Tasa & Defunciones & Tasa \\
\hline 1955 & 35 & 1,1 & 27 & 0,8 & 62 & 0,9 & 1979 & 111 & 2,1 & 31 & 0,6 & 142 & 1,3 \\
\hline 1956 & 37 & 1,1 & 23 & 0,6 & 60 & 0,9 & 1980 & 95 & 1,7 & 32 & 0,6 & 127 & 1,1 \\
\hline 1957 & 40 & 1,1 & 15 & 0,4 & 55 & 0,8 & 1981 & 94 & 1,7 & 30 & 0,5 & 124 & 1,1 \\
\hline 1958 & 55 & 1,5 & 22 & 0,6 & 77 & 1,1 & 1982 & 124 & 2,2 & 27 & 0,5 & 151 & 1,3 \\
\hline 1959 & 45 & 1,2 & 25 & 0,7 & 70 & 0,9 & 1983 & 115 & 2,0 & 18 & 0,3 & 133 & 1,1 \\
\hline 1960 & 45 & 1,2 & 30 & 0,8 & 75 & 1,0 & 1984 & 105 & 1,8 & 29 & 0,5 & 134 & 1,1 \\
\hline 1961 & 54 & 1,4 & 32 & 0,8 & 86 & 1,1 & 1985 & 119 & 2,0 & 37 & 0,6 & 156 & 1,3 \\
\hline 1962 & 53 & 1,3 & 23 & 0,6 & 76 & 0,9 & 1986 & 90 & 1,5 & 37 & 0,6 & 127 & 1,0 \\
\hline 1963 & 56 & 1,4 & 25 & 0,6 & 81 & 1,0 & 1987 & 101 & 1,6 & 42 & 0,7 & 143 & 1,1 \\
\hline 1964 & 72 & 1,7 & 27 & 0,6 & 99 & 1,2 & 1988 & 113 & 1,8 & 44 & 0,7 & 157 & 1,2 \\
\hline 1965 & 56 & 1,3 & 18 & 0,4 & 74 & 0,9 & 1989 & 102 & 1,6 & 38 & 0,6 & 140 & 1,1 \\
\hline 1966 & 64 & 1,5 & 28 & 0,6 & 92 & 1,1 & 1990 & 114 & 1,8 & 41 & 0,6 & 155 & 1,2 \\
\hline 1967 & 58 & 1,3 & 21 & 0,5 & 79 & 0,9 & 1991 & 104 & 1,6 & 44 & 0,7 & 148 & 1,1 \\
\hline 1968 & 71 & 1,6 & 31 & 0,7 & 102 & 1,1 & 1992 & 113 & 1,7 & 36 & 0,5 & 149 & 1,1 \\
\hline 1969 & 58 & 1,3 & 34 & 0,7 & 92 & 1,0 & 1993 & 130 & 1,9 & 39 & 0,6 & 169 & 1,2 \\
\hline 1970 & 76 & 1,7 & 32 & 0,7 & 108 & 1,2 & 1994 & 114 & 1,6 & 46 & 0,7 & 160 & 1,1 \\
\hline 1971 & 62 & 1,4 & 28 & 0,6 & 90 & 1,0 & 1995 & 143 & 2 & 49 & 0,7 & 192 & 1,4 \\
\hline 1972 & 75 & 1,6 & 27 & 0,5 & 102 & 1,1 & 1996 & 100 & 1,4 & 37 & 0,5 & 137 & 1,0 \\
\hline 1973 & 85 & 1,8 & 34 & 0,7 & 119 & 1,2 & 1997 & 109 & 1,4 & 43 & 0,5 & 152 & 1,0 \\
\hline 1974 & 97 & 2,0 & 41 & 0,8 & 138 & 1,4 & 1998 & 117 & 1,6 & 42 & 0,6 & 159 & 1,1 \\
\hline 1975 & 99 & 2,0 & 26 & 0,5 & 125 & 1,2 & 1999 & 125 & 1,7 & 47 & 0,6 & 172 & 1,1 \\
\hline 1976 & 90 & 1,8 & 36 & 0,7 & 126 & 1,2 & 2000 & 139 & 1,8 & 45 & 0,6 & 184 & 1,2 \\
\hline 1977 & 94 & 1,8 & 26 & 0,5 & 120 & 1,1 & 2001 & 134 & 1,7 & 48 & 0,6 & 182 & 1,2 \\
\hline 1978 & 93 & 1,8 & 22 & 0,5 & 115 & 1,1 & 2002 & 139 & 1,9 & 54 & 0,7 & 193 & 1,3 \\
\hline
\end{tabular}




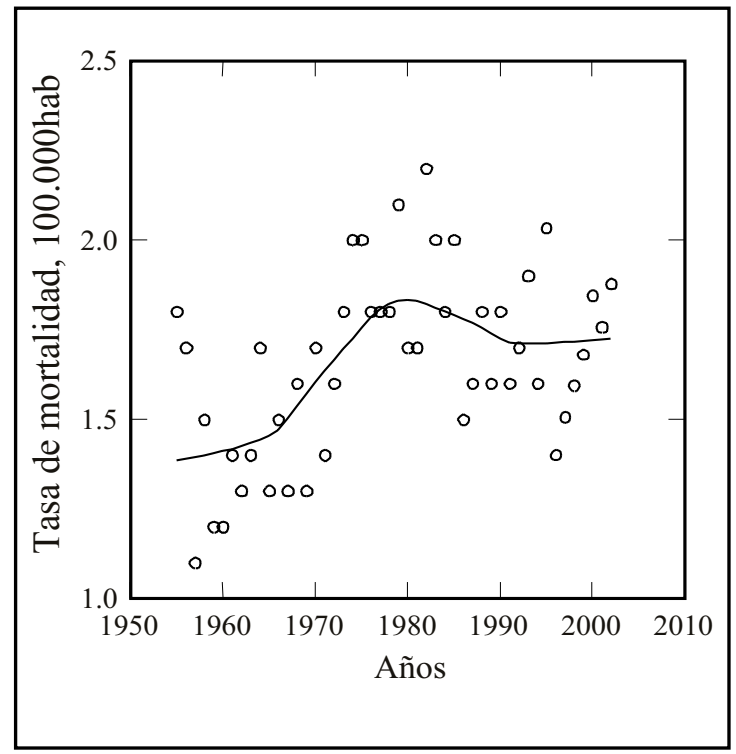

Figura 2. Tasa bruta de mortalidad por cáncer oral y faríngeo en hombres. 1955-2002.

La mortalidad agrupada por edad y género de 1982 a 2002 para el cáncer oral y faríngeo, afectó en $74 \%$ a hombres y $26 \%$ a mujeres (H:M 2,8:1). En hombres se encontró el mayor número de defunciones entre los 55 y 64 años (30,5\%), y disminución en los mayores de 75 años $(18,4 \%)$. En cambio, en mujeres el mayor número de casos (42,9\%) ocurrió después de los 75 años (Figura 4).

Por ubicación anatómica, para el período 1982 al 2002, el sitio más frecuente de mortalidad por

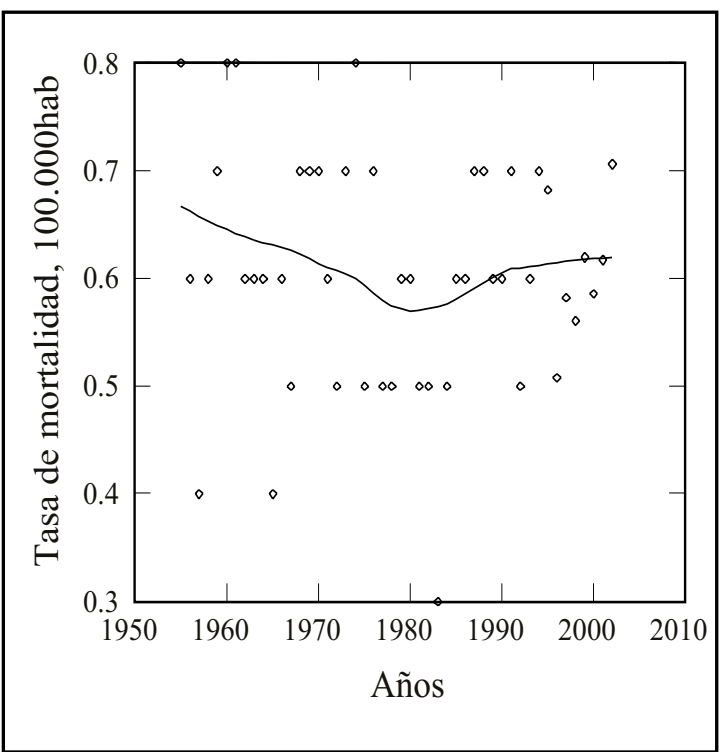

FIgURA 3. Tasa bruta de mortalidad por cáncer oral y faríngeo en mujeres. 1955-2002.

cáncer oral fue lengua, representó el 39\%, seguido por glándulas salivales mayores $30 \%$, piso de boca $18 \%$, labio $9 \%$, y encía $4 \%$.

Para el cáncer de lengua la relación H:M fue 3,6:1. Los hombres presentaron el mayor número de casos entre los 55 y 64 años $(33,6 \%)$, y disminuyó a los 75 años (13\%). En cambio para mujeres, el número de casos aumentó progresivamente con la edad, observándose el mayor número de defunciones después de los 75 años (39,6\%) (Figura 5).

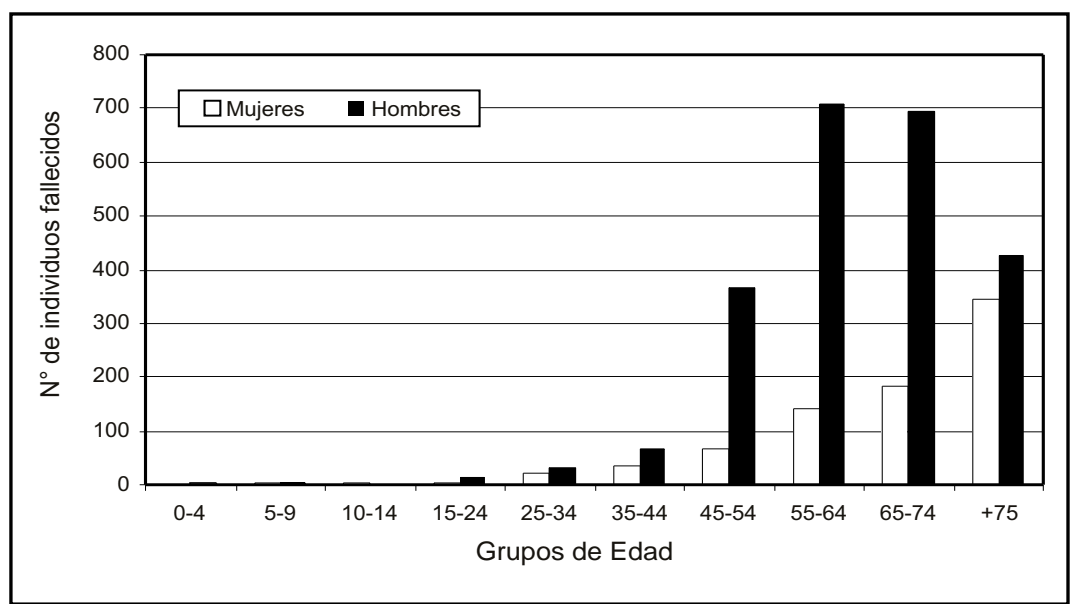

Figura 4. Mortalidad agrupada por edades, para cáncer oral y faríngeo, por género. 1982-2002. 


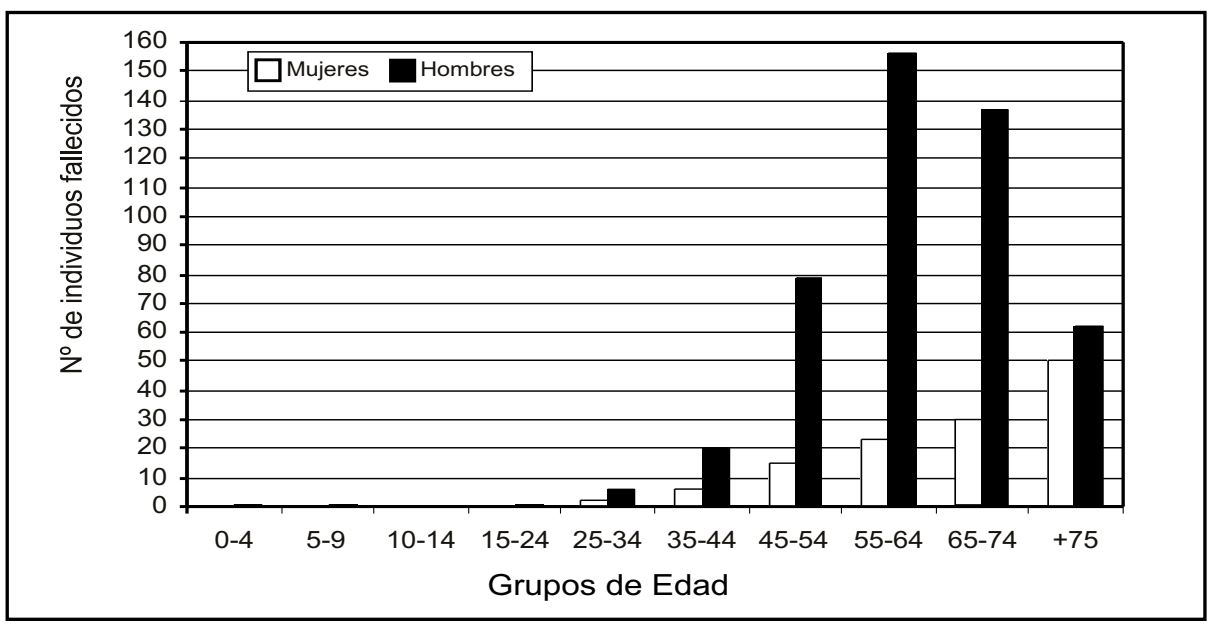

FiguRA 5. Mortalidad agrupada para cáncer de lengua, por géneros. 1982-2002.

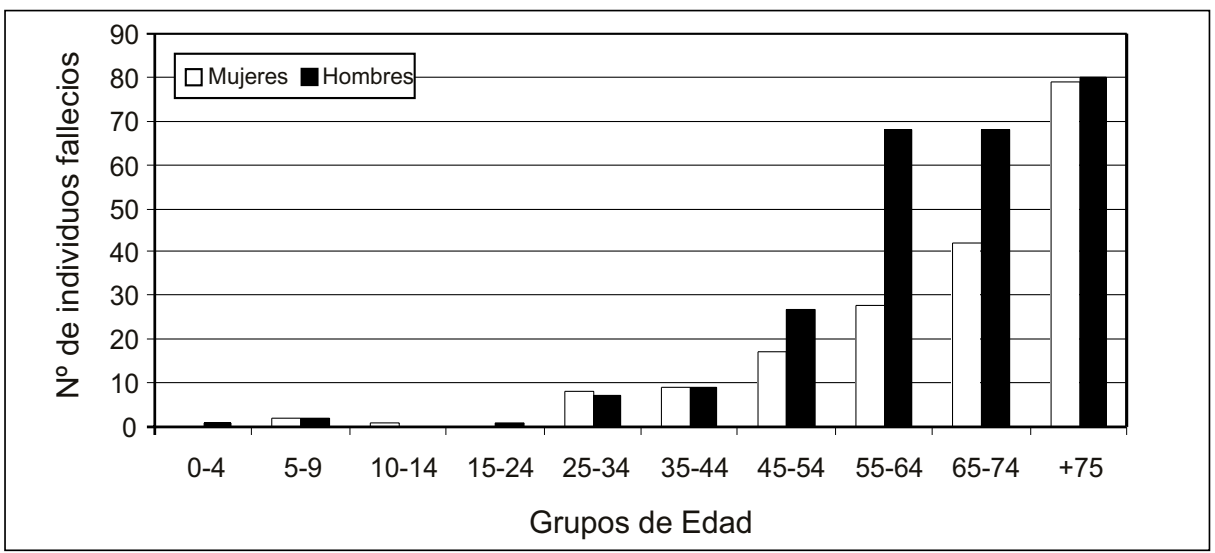

Figura 6. Mortalidad agrupada para cáncer de glándulas salivales mayores, por géneros. 1982-2002.

En cáncer de glándulas salivales mayores, la mortalidad fue aumentando progresivamente con la edad, tanto en hombres como en mujeres y el mayor número de defunciones ocurrió en los mayores de 75 años (35,4\%). En este cáncer es donde se observan las menores diferencias por géneros, la razón H:M fue de 1,4:1 (Figura 6).

Para el cáncer de piso de boca, la razón H:M fue 4:1, en esta ubicación observamos las mayores diferencias por género. En hombres, principales afectados, el mayor número de defunciones se produjeron entre los 55-64 años (33,3\%) mientras que en mujeres, la mortalidad fue en aumento junto con la edad, observándose la mayor mortalidad en las mayores de 75 años (41,8\%), sin embargo, para este grupo de edad se acortan las diferencias por géneros $(55,7 \%$ hombres y $44,3 \%$ en mujeres) (Figura 7).

Morbilidad. La morbilidad por cáncer oral y faríngeo representó, aproximadamente, el 1,6\% con respecto al total de cánceres. Para el cáncer oral y faríngeo la razón H:M fue 2,3:1. Al analizar la tasa bruta de morbilidad para los años 1969 a 2002 observamos 


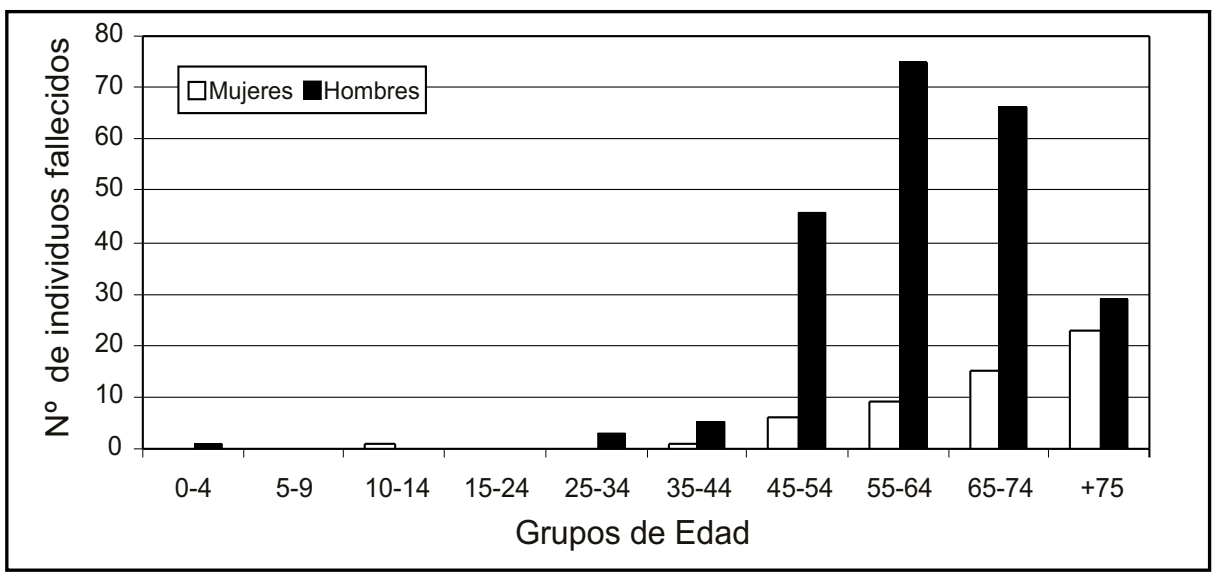

Figura 7. Mortalidad agrupada por cáncer de piso de boca, ambos géneros. 1982-2002. (No incluye año 1993 por error en la publicación original).

que aumentó en 79,9\% anual (Tabla 2, Figura 8). Por géneros, observamos que afectó a $70 \%$ de los hombres y a 30\% de mujeres (H:M 2,3:1), en hombres se produjo un aumento de la morbilidad después de los 45 años, para llegar al máximo número de casos en los 55-64 años (34,2\%), disminuyendo luego con la edad. En mujeres se observó

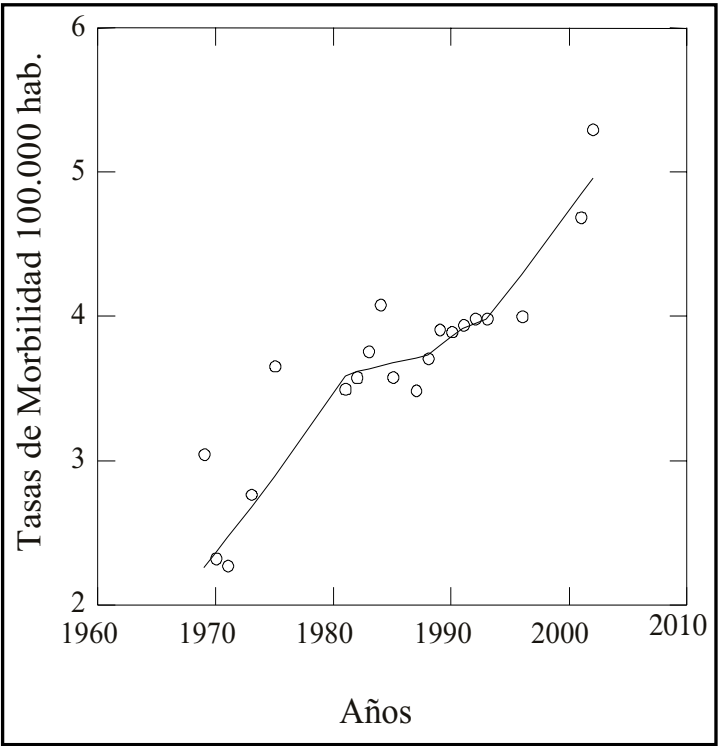

Figura 8. Tasa de morbilidad por cáncer oral y faríngeo, ambos géneros. 1969 2002. (Sin información años 1972, 1974, desde 1976 a 1981, 1986, 1994 , 1996 y desde 1997 a 2000). aumento progresivo de la morbilidad, el mayor número de casos ocurrió en el grupo de los 65-74 años (22,2\%), y se produjo leve disminución en las mayores de 75 años (21,1\%), (Figura 9).

Tabla 2. M orbilidad por cáncer oral y faríngeo, tasa bruta de morbilidad, ambos géneros, Chile 19812002. (Sin información los años 1972, 1974, 19761980, 1986, 1994, 1995 y desde 1997 a 2000)

\begin{tabular}{|lcc|}
\hline Año & $N^{\circ}$ Casos & Tasa bruta \\
\hline 1969 & 284 & 3 \\
1970 & 215 & 2,3 \\
1971 & 214 & 2,3 \\
1973 & 270 & 2,8 \\
1975 & 373 & 3,7 \\
1981 & 395 & 3,5 \\
1982 & 411 & 3,6 \\
1983 & 439 & 3,8 \\
1984 & 485 & 4,1 \\
1985 & 431 & 3,6 \\
1987 & 437 & 3,5 \\
1988 & 473 & 3,7 \\
1989 & 507 & 3,9 \\
1990 & 510 & 3,9 \\
1991 & 525 & 3,9 \\
1992 & 540 & 4,0 \\
1993 & 549 & 4,0 \\
1996 & 577 & 4,0 \\
2001 & 722 & 4,7 \\
2002 & 797 & 5,3 \\
\hline
\end{tabular}




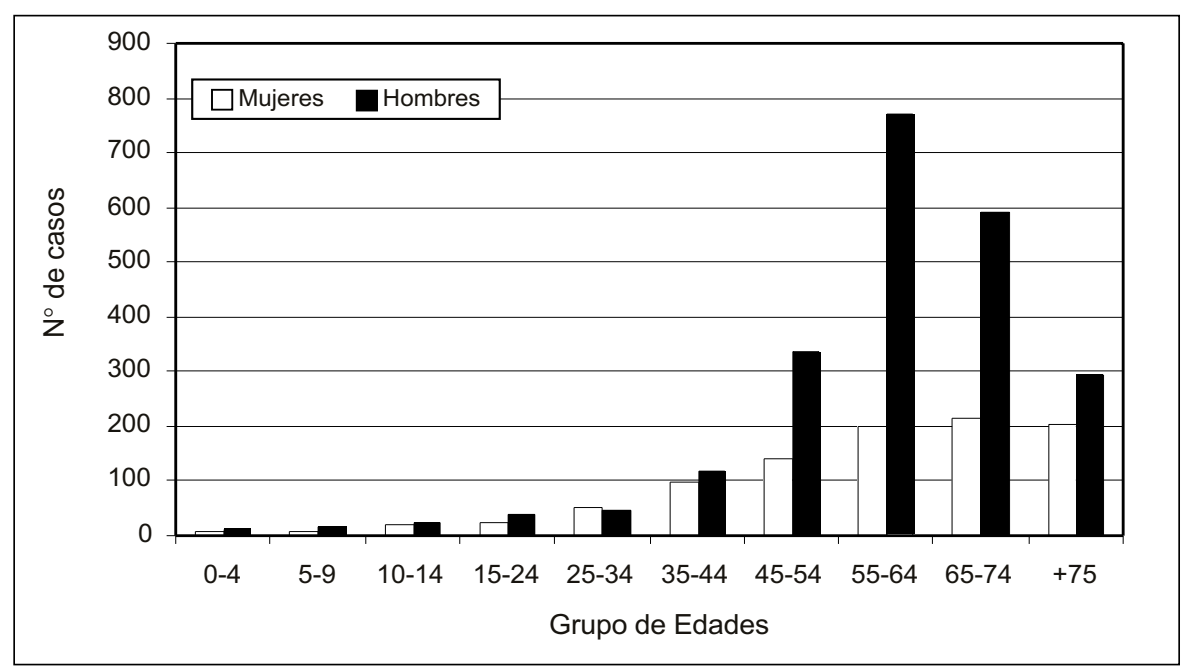

Figura 9. Morbilidad agrupada, para cáncer oral y faríngeo, por género. 19891997. (Sin información años 1994, 1995 y desde 1997 al 2000, excluidos años 20012002).

DisCUSIÓN

La morbilidad por cáncer oral y faríngeo en Chile correspondió aproximadamente a 1,6\% del total de cánceres, esta cifra es menor a lo que se observa en otros países, tales como Estados Unidos donde la incidencia es entre 2-3\% ${ }^{12}$, mientras que en India representa cerca de $20 \% 13$. Estos resultados se asocian a exposición a factores de riesgo, como consumo de alcohol y tabaco en sus distintas presentaciones (pipas, masticar tabaco, uso de snuff $)^{3,14,15}$. En Chile no existe la costumbre de masticar tabaco pero $48,7 \%$ de la población es fumadora ${ }^{16,17}$ y 93\% del cáncer oral se asocia al tabaco ${ }^{18}$.

Es preocupante que la morbilidad por cáncer oral y faríngeo experimentó un aumento progresivo de la tasa de 2,3 puntos desde 1969 a 2002. La razón $\mathrm{H}: \mathrm{M}$ observada fue de 2,3:1, encontrándonos a un nivel muy similar con lo que ocurre a nivel mundial. En 1950, en Estados Unidos, la relación $\mathrm{H}: \mathrm{M}$ era de $6: 1$, pero el aumento del consumo de tabaco en mujeres en las últimas tres décadas, produjo una mayor incidencia de cáncer oral, modificándose la relación H:M a 2:19,20. Lo cual también está ocurriendo en Chile para el cáncer oral. Todo indica que deben incrementarse las medidas preventivas a nivel escolar, especialmente, en el grupo femenino donde se está observando un importante aumento de morbilidad por cáncer oral.
El $84,4 \%$ de los casos en Chile se produjo después de los 45 años y, tanto en hombres como en mujeres, la morbilidad fue aumentando progresivamente con la edad, pero disminuyó en los mayores de 75 años; en hombres el mayor número de casos se observó entre los 55 y 64 años, mientras que en mujeres, el mayor número de casos se observó entre los 65 y 74 años. Numerosos estudios demuestran que el mayor número de casos de cáncer oral se produce después de los 45 años, siendo le edad media los 64 años $^{20}$; Silverman et al (2001) encontraron 90\% de los casos de cáncer oral después de los 45 años, siendo la edad promedio de diagnóstico después de los 60 años $^{5}$. Miller et al (1996), en Estados Unidos, encontraron que la incidencia por cáncer oral aumentó con la edad para todos los grupos en ambos géneros, excepto para el grupo de más de 70 años ${ }^{2}$.

Chile no cuenta actualmente con registros sobre incidencia ni prevalencia para cáncer a nivel nacional, pero se están haciendo esfuerzos para lograr mejorar esta información y, desde 1993, se está registrando en Valdivia, desde 1998 en Antofagasta y desde 2003 en Concepción ${ }^{22}$, esperamos que este registro pueda incluir a futuro a todo el país ya que es fundamental conocer las tendencias que sigue el cáncer. La mortalidad por cáncer oral y faríngeo en el mundo corresponde aproximadamente al 4,4\% del total de cánceres ${ }^{23}$. En Chile correspondió a 1\% dentro del total de casos de 
cánceres, pero al observarse un aumento de morbilidad, especialmente en mujeres, es posible esperar un incremento en la mortalidad en los próximos años si no se toman medidas adecuadas de prevención.

La tasa bruta de mortalidad por cáncer oral y faríngeo en Chile aumentó desde 1955 hasta 1980. Sin embargo, para el período 1981 a 2002, la tasa se estabilizó manteniéndose relativamente constante, existiendo incluso una leve disminución $(0,01 \%$ anual). En Estados Unidos, la mortalidad por cáncer oral y faríngeo ha ido disminuyendo desde 1970, obteniendo mejores niveles de supervivencia, esto lo asocian a un mayor conocimiento acerca del cáncer, campañas de prevención, mejor tecnología y diagnóstico precoz ${ }^{24,25}$.

Si se compara la mortalidad para cáncer oral y faríngeo en Chile por género, encontramos que los principales afectados fueron los hombres $(74 \%$ versus $26 \%$ en mujeres) lo cual es similar a la mayoría de los países ${ }^{5,25}$, debido a la mayor frecuencia del hábito de fumar asociado a alta ingesta de alcohol.

En hombres, la tasa de mortalidad mostró aumento desde 1955 hasta 1980 (tasa: 1,1 a 2,1/ 100.000 hab), posteriormente se observó una disminución desde 1981 a 1991 (tasa de 2,1 a 1,6/ 100.000 hab), para estabilizarse y mantenerse relativamente constantes hasta 2002. En mujeres se observó un fenómeno opuesto, la tasa de mortalidad disminuyó desde 1955 hasta 1980 (tasa: 0,8 a 0,6/100.000 hab), mientras que desde 1981 a 2002 se observó aumento en la tasa (tasa: 0,5 a $0,7 / 100.000$ hab). Esto podría asociarse a los cambios en el estilo de vida que han experimentado las mujeres en los últimos años, como es el tabaco, ya que aumentaron su consumo en más

\section{REFERENCIAS}

1. Milián A, González M. Tumores malignos de la mucosa oral. En: Bagán (Ed.) Medicina Oral. Barcelona-España, editorial Masson, 1995; 18799.

2. Miller B, Bernstein L, Young L, Swanson GM, West D, Liff JM et AL. Oral Cavity: US Racial/ethnic Cancer Patterns 1992, National Cancer Institute de 100\% entre 1970 y 1998, mientras que los hombres lo hicieron sólo en 13\%16,26,27.

$\mathrm{Al}$ analizar lo que ocurrió con la mortalidad por cáncer oral en los distintos grupos de edad, encontramos que $94 \%$ de las defunciones ocurrieron en los mayores de 45 años, estos datos concuerdan con los encontrados por Kischbaum y col (1986). En hombres el mayor número de defunciones se producen entre los 55-64 años, mientras que en mujeres se producen después de los 75 años $^{28}$.

En Chile, desde 1982 a 2002, el cáncer de lengua (39\%) fue el que provocó mayor mortalidad dentro del cáncer oral, le siguieron cáncer de glándulas salivales mayores (30\%), cáncer de piso de boca (18\%), de labio (9\%), y, por último, el que provocó las menores defunciones fue el cáncer de encía (4\%). Kischbaum y col (1986) encontraron la mayor prevalencia para cáncer de lengua, en segundo lugar el cáncer bucofaríngeo y en tercer lugar el cáncer de glándulas salivales ${ }^{28}$. El cáncer de borde de lengua es el más frecuentemente diagnosticado en Chile, por lo tanto, el médico y el dentista deben examinar adecuadamente el borde de lengua, en busca de lesiones cancerizables, tales como leucoplasias u otras, o detectar tempranamente la lesión maligna.

El cáncer de lengua es el más común en muchos países ${ }^{28,30}$, en Chile la mayor mortalidad por este cáncer ocurrió en hombres, encontrándose una relación H:M 3,6:1; la edad donde se produjo el mayor número de defunciones fue entre 55 y 64 años en hombres, mientras que en mujeres fue en mayores de 75 años. En Estados Unidos la edad promedio de fallecimiento por este tipo de cáncer fue de 67 años, 64 años en hombres y 71 años en mujeres, y al igual que en Chile mueren a una edad mayor $^{31}$. Por lo tanto, lesiones iniciales deben empezar a buscarse después de los 40 años.

EEUU. Disponible en: www.cancer.gov [Consultado el 9 de enero de 2004].

3. Johnson N, Bain C, and CO-Authors of the EUWorking Group on Tobacco and Oral Health. Tobacco And Oral Disease. Br DentJ 2000; 189: 200-6.

4. Gillison ML, Koch W, Capone R, Spafford M, Westra WH, Wu L ET AL. Evidence for a causal association between human papillomavirus and a subset of head and neck cancers. J Natl Cancer Inst 2000; 92: 709-20. 
5. Silverman S. Demographics and ocurrence of oral and pharyngeal cancers, the outcomes, the trends, the challenge. JADA 2001; 132: 7-11.

6. National CANCer Institute. Nasopharyngeal Cancer, 2003. Disponible en: www.cancer.gov [Consultado el 10 de enero de 2004].

7. National Cancer Institute. Hypopharyngeal Cancer; 2003. Disponible en: www.cancer.gov [Consultado el 10 de enero de 2004].

8. Organización Panamericana de la Salud, OPS. De Datos Básicos a Índices Compuestos: Una Revisión del Análisis de Mortalidad Boletín Epidemiológico 200; 23(4), Disponible en: www.paho.org [Consulta el 12 de enero de 2004].

9. Ministerio de Salud Chile. Estadísticas en Salud, Departamento de estadística e información de salud.

10. Instituto Nacional de Estadísticas Chile, Anuario de estadísticas vitales y anuario de demografía.

11. Wilkinson L. Systat. V10.2. Chicago, III. USA. 2002.

12. Casiglia J, Woo B. A comprehensive review of oral cancer. Gen Dent 2001; 49: 72-82.

13. Balaram P, Meenattoor G. Immunology of oral cancer a review Singapore Dent J 1996; 21: 36-41.

14. Gupta PC, Ray CS. Smokeless tobacco and health in India and South Asia. Respirology 2003; 8: 419-31.

15. Neville B, Damm D, Allen C, Bouquot J. Oral \& Maxillofacial Pathology, USA, Saunders Company: 1995; 295-7.

16. Alvarado R, Talavera G. La epidemia de tabaquismo en Chile: evolución durante la última década. Rev Chil Salud Pública 2003; 7: 69-73.

17. Ministerio de Salud. Promoción de una vida sin tabaco; Minsal Depto. epidemiología el vigía $\mathrm{n}^{\circ} 15$. http://epi.minsal.cl/evigia/

18. Organización Mundial de la Salud, Chile. Diagnóstico epidemiológico del tabaquismo, daños producidos por el tabaco y costos económicos. http:/ /pwr-chi.bvsalud.org

19. Center for Disease Control and Prevention (CDC). Preventing and Controlling Oral and Pharyngeal
Cancer Recommendations from a National Strategic Planning Conference 1998. www.cdc.gov

20. National Cancer Institute. Realidades del cáncer bucal 2004. www.cancer.gov

21. Center for Disease Control and Prevention (CDC). Improving diagnoses of oral cancer Chronic Disease Notes \& Reports 2000; 13(3). www.cdc.gov

22. Vallebuona C. Registros Poblacionales de Cáncer: Avance en Chile, Depto. Epidemiología http:// epi.minsal.cl/evigia/

23. Organización Mundial de la Salud (OMS). Informe sobre la salud en el mundo 2003. Defunciones por causa, sexo y estratos de mortalidad en las regiones de la OMS. Www.cancer.org

24. National Cancer Institute. Progress Shown in Death Rates From Four Leading Cancers 2003. www.cancer.org

25. American Cancer Society. Cancer Facts \& Figures 2004. WWW.cancer.org

26. American Cancer Society. Detailed Guide: Oral Cavity and Oropharyngeal Cancer What are The Key Statistics About Oral Cavity and Oropharyngeal Cancer 2003. www.cancer.org

27. National Cancer Institute. What are the key statistics about oral cavity and oropharygeal cancer; 2003. Www.cancer.gov

28. Kirschbaum A, Vincent M, Kohen M. Tendencia de la mortalidad por cáncer bucofaríngeo en Chile sexenio 1980-1985. Rev Fac Odont Univ de Chile 1988; 6: 16-9.

29. Moore SR, Johnson NW, Pierce AM, Wilson DF. The epidemiology of tongue cancer: a review of global incidence. Oral Dis 2000; 6: 75-84.

30. Shiboski CH, Shiboski SC, Silverman S Jr. Trends in oral cancer rates in the United States, 19731996. Community Dent Oral Epidemiol 2000; 28: 249-56.

31. National Cancer Institute. SEER Cancer Statistics Review 1975-2000, Age distribution of deaths by site, all races, both sexes 1996-2000 Table I-12. http://seer.cancer.gov

\section{Agradecimientos}

Expresamos nuestros sinceros agradecimientos al personal del Centro de Documentación del Ministerio de Salud y del Instituto Nacional de Estadística, quienes siempre estuvieron dispuestos a colaborar con información para este estudio. 